\title{
Ubi caritas et amor. Une lecture comparée du Baiser au lépreux et de $\mathrm{O}$ Veleiro de Cristal
}

\author{
SAMUEL BIDAUD
}

\begin{abstract}
Ubi caritas et amor. A Comparatist Reading of Le Baiser au lépreux and $\mathrm{O}$ Veleiro de Cristal. Le Baiser au lépreux (The Kiss to the Leper), by François Mauriac, and O Veleiro de Cristal (The Crystal Sailboat), by José Mauro de Vasconcelos, are a priori aimed at two different audiences. Le Baiser au lépreux is indeed generally considered to be Mauriac's first important book, whereas $O$ Veleiro de Cristal belongs to "children's literature", like O Meu Pé de Laranja Lima (in English "My Sweet Orange Tree"), by the same author, which was very successful in Brazil but also in France. However, the two stories share deep similarities, so we can draw parallels between them. As far as the narrative level is concerned, both of the books tell the story of a human being who suffers because of his physical appearance; Jean Péloueyre in Le Baiser au lépreux and Eduardo in $O$ Veleiro de Cristal are rejected by their close relatives and hide from other people. They try to escape from their painful experience, thanks to the religion in the case of Jean Péloueyre, even if the latter gives rise to an existential drama, and thanks to the imagination in the case of Eduardo, who discovers in the house where his aunt Anna has led him animals who are able to talk. In both stories, eventually, the theme of charity is fundamental: in Mauriac's novel, Noémi marries Jean and ends up loving him with a love based on charity and that can be defined as agapè, while in O Veleiro de Cristal Anna renounces all vanity to devote herself to her nephew, her charity echoing the one of the animals who live in the Crystal Sailboat.
\end{abstract}

Keywords: Le Baiser au lépreux; François Mauriac; O Veleiro de cristal; José Mauro de Vasconcelos

\section{Introduction}

Peut-être pour avoir lu le premier et traduit le second à peu près au même moment, l'idée de comparer Le Baiser au lépreux de François Mauriac (1969 [1922]) et O Veleiro de Cristal (français « Le Voilier de Cristal ») de José Mauro de Vasconcelos (1973) s'est imposée à nous comme une évidence ${ }^{1}$, et ceci, en

1 Notre traduction de $O$ Veleiro de Cristal est en cours d'évaluation. 
dehors de toute influence d'une œuvre sur l'autre - car, entre les livres de Mauriac et de José Mauro de Vasconcelos, qui se sont probablement ignorés, les différences sont grandes. Plusieurs points rapprochent toutefois les deux récits sur lesquels nous nous pencherons ici. Leur histoire, d'abord : celle de deux êtres souffrants de leur apparence physique, Jean Péloueyre dans Le Baiser au lépreux et Eduardo dans $O$ Veleiro de Cristal, qui luttent contre leur physique ingrat et leur infirmité, lesquels entraînent leur rejet et leur mort. La charité qui vainc la mort ensuite : représentée dans Le Baiser au lépreux par le personnage de Noémi, qui épouse Jean Péloueyre et finit par l'aimer, mais aussi par Jean Péloueyre lui-même, et par Anna dans $O$ Veleiro de Cristal, qui est entièrement dévouée à son neveu malade, elle brille comme la lumière de l'espérance dans les deux récits, par-delà la tristesse et la souffrance qu'ils disent.

\section{Quelle laideur?}

Jean Péloueyre et Eduardo, les personnages principaux du Baiser au lépreux et de $O$ Veleiro de Cristal, ressentent un sentiment de honte à l'égard de leur apparence physique. Cette dernière est à l'origine de leur rejet non seulement par les autres, mais également par eux-mêmes.

Le rejet dont Jean Péloueyre est victime est manifeste dans Le Baiser au lépreux. Il se reflète, à un premier niveau, dans le double exil auquel Jean est contraint pour se cacher des autres à qui il inspire du dégoût. Au début du récit, Jean cherche continuellement à se dérober au regard d'autrui et se réfugie dans la campagne ou à l'église, là où il est sûr de ne pas être vu et de se trouver à l'abri des moqueries. Cette première forme de rejet est due à l'image que les autres ont de Jean et que ce dernier a intériorisée. La situation évolue après qu'il a épousé Noémi : alors que, au début, Jean fuit de lui-même et par sa propre volonté, cette fois ce sont ses proches qui l'encouragent, pour ne pas dire qui l'obligent, à partir. En effet, le curé qui est à l'origine du mariage de Jean et Noémi voit que la jeune femme dépérit en vivant aux côtés de Jean, et que, malgré tous les efforts qu'elle fait, la présence de son mari la rend malade. Jean est ainsi éloigné à Paris sous le prétexte de lui faire écrire un livre qu'il ne veut pas vraiment écrire : il se produit donc un second rejet, inverse celuilà, puisque Jean n'en est plus l'agent mais le patient. On note que ce double rejet, actif puis passif, envoie Jean dans deux lieux opposés : la campagne et l'église solitaires lorsque c'est lui qui part de lui-même dans un premier temps, la ville immense qu'est Paris, au contraire, lorsqu'on l'éloigne en forçant plus ou moins sa décision dans un second temps ; cette double dimension du rejet, actif et passif, vers un lieu où il est seul aussi bien que vers la foule, révèle bien le 
caractère quasi intégral de l'exil de Jean, repoussé partout et qui semble n'avoir le droit d'être nulle part.

À Paris, toutefois, survient un changement dans la perception qu'a Jean de la laideur : il découvre également celle-ci peinte sur les autres visages, au point qu'il se fond dans une foule où il passe désormais inaperçu (Mauriac 1969 : 108-109). En réalité, cette laideur n'est pas que physique ; elle est celle d'un mal-être profond, celui de la civilisation anonyme :

Jean s'installa, chaque après-midi, à la terrasse du café de la Paix, au bord d'un triste fleuve de visages. Les maladies secrètes, l'alcool, les stupéfiants avaient repétri à il ne savait quelle immonde ressemblance des milliers de figures qui toutes furent des figures d'enfants. (Ibid. 111)

La laideur se fait ainsi existentielle : c'est celle de l'absence de toute forme de vraie joie et de vie intérieure. Le contraste entre le « triste fleuve de visages » et « qui furent des figures d'enfants » est saisissant : ce qu'oppose ici Mauriac, c'est la vie en surface, à la dérive, de ces personnes dont le sentiment de vie et de pureté semble s'être éloigné, et l'enfant qu'elles ont été (le passé simple, « qui toutes furent des figures d'enfants », semble rejeter cette époque dans un passé presque mythique et lointain); Mauriac dit ainsi le drame de ces êtres qui se laissent emporter par la vie et comme sombrer, oubliant qu'ils ont une âme celle que l'enfant qu'il y a en eux continue à porter, secrètement, en attendant qu'ils la laissent s'éveiller de nouveau.

Rappelons enfin la présence récurrente de l'objet du miroir dans Le Baiser au lépreux. Le roman de Mauriac s'ouvre sur une scène où Jean Péloueyre se regarde dans une glace et se fuit (Ibid. 11-12), et le motif du miroir se retrouve aussi à la fin du récit, lorsque Jean est mort : « Les miroirs, où si souvent Jean Péloueyre avait contemplé sa pauvre mine, furent voilés de linge » (Ibid. 171). Cette présence du miroir, à la fois au tout début et à la fin du récit, c'est-àdire à deux moments-clés, est révélatrice. On passe d'un miroir qui reflète Jean à des miroirs voilés. Alors que Jean est, au début, prisonnier de l'image qu'il renvoie aux autres et à lui-même, et donc prisonnier d'une dimension corporelle, les miroirs voilés évoquent, au contraire, l'accès à une vie qui ne peut plus être vue physiquement, mais qui est la vraie vie à laquelle la mort donne accès. Il n'y a plus de miroir pour refléter, car il n'y a plus rien de visible à refléter. Seul demeure l'essentiel, l'invisible qu'on ne voit bien qu'avec le cœur, pour paraphraser Saint-Exupéry. Le miroir n'est pas un objet de vérité mais de mensonge dans le roman de Mauriac; il ne montre qu'une apparence derrière laquelle se trouve l'âme, la réalité la plus profonde de la personne pour le chrétien qu'est Mauriac. 
BIDAUD

Le thème du rejet dû aux apparences est également présent dans $O$ Veleiro de Cristal. Eduardo souffre en effet d'une maladie qui le déforme physiquement : il a, notamment, une tête énorme. Il éprouve par ailleurs de très grandes difficultés à marcher, et ne peut se déplacer qu'à l'aide de béquilles. Son apparence provoque le dégoût des autres : les visiteurs qui viennent voir sa famille disent qu'ils ont peur de rencontrer «le petit monstre ${ }^{2} \gg$ (Vasconcelos 1973 : 37). Eduardo, comme Jean Péloueyre, a intériorisé un sentiment de honte vis-à-vis de son apparence physique ; il se met de lui-même à l'écart lorsqu'il y a du monde. Il est rejeté par sa famille : cette dernière le cache aux visiteurs, lui préférant ses deux frères en bonne santé, Marcelo et Sérgio, et elle ne se préoccupe guère de lui. Lorsqu'il se retrouve dans un hôpital pour les enfants souffrant d'un handicap mental, personne ne vient l'en sortir, et tous ses efforts pour dire au personnel qu'il n'est pas comme les autres enfants restent vains - on ne le laisserait pas rentrer chez lui, si sa tante Anna ne faisait mille efforts pour l'aider. Dans cet hôpital il découvre des enfants abandonnés dont les parents ont honte :

Il y avait là-bas deux cents malades. Soixante d'entre eux, dont les mères avaient honte et qu'elles ne venaient chercher qu'une fois la nuit tombée, quand personne ne pouvait plus voir leur malheur. Les cent quarante autres étaient d'autres mères qui ne voulaient plus entendre parler de la monstruosité de leurs enfants. Comme c'était triste. (Ibid. 43)

Eduardo est toutefois animé par un désir de transformation physique qui lui permettrait, pense-t-il, d'obtenir l'affection tant désirée de sa famille. Or cette transformation physique survient une nuit, lorsque Gakusha, le tigre qui parle, permet à Eduardo de retrouver le temps d'une promenade des jambes normales. Eduardo voudrait alors pouvoir se regarder dans une glace, mais Gakusha s'y oppose : les miroirs, affirme ce dernier, sont les pires ennemis des illusions et des rêves. L'objet du miroir est ici aussi dévalorisé, comme dans Le Baiser au lépreux, dans le sens où il ne reflète pas la vraie personne qui se définit par son intériorité (par exemple, dans le cas d'Eduardo, par sa capacité à rêver), mais la personne dans son aspect de surface. Le vrai est avant tout situé dans l'imagination pour Eduardo.

José Mauro de Vasconcelos reprend, avec le personnage d'Eduardo, l'opposition chère à Victor Hugo de la laideur extérieure et de la beauté intérieure (que l'on pense à Quasimodo dans Notre-Dame de Paris). Anna affirme ainsi

2 C'est nous qui traduisons, ici comme dans les autres citations ; pour des raisons d'espace, nous ne reproduisons pas le texte original en portugais. 
à Eduardo : « Tu as un cœur qui est excessivement beau. Et il se trouve que peu de gens l'ont découvert » (Ibid. 22). Quant à Anna elle-même, elle a renoncé à sa propre beauté extérieure, qui l'entraînait vers la futilité, pour découvrir sa beauté intérieure et l'amour du prochain auprès de son neveu - en d'autres termes, c'est là, dans cette beauté toute intérieure qui n'est autre que la vraie beauté et qui renvoie à l'amour en tant qu'agapè, qu'elle et Eduardo se rencontrent.

L'opposition de l'apparence et de l'intériorité, du visible et de l'invisible, se retrouve chez les animaux qui habitent le Voilier de Cristal, nom dont Eduardo a baptisé la maison où il est parti se reposer avec sa tante Anna. Ceuxci paraissent a priori dépourvus de vie : Gakusha le tigre, qui deviendra l'ami d'Eduardo, lui apparaît sous la forme d'une statue de bronze, et Maria Jurandir sous celle d'une chouette empaillée. Or tous deux sont vivants et s'animent à certains moments. Gakusha quitte ainsi son enveloppe corporelle de tigre de bronze pour rejoindre Eduardo une nuit où il l'emmène voyager sur son dos, et, comme ce dernier lui exprime sa surprise qu'il ait perdu la dureté de cuivre de la statue, Gakusha explique : «Cette dureté est restée là-bas, dans l'autre [tigre]. Je suis en quelque sorte son âme » (Ibid. 60).

On note enfin que l'histoire d'Eduardo est redoublée, dans $O$ Veleiro de Cristal, par celle de la chouette Maria Jurandir. Celle-ci lui confie en effet que lorsqu'elle est née, elle et son frère ont suscité les moqueries de l'ensemble des visiteurs qui sont venus voir sa mère. Mais, contrairement à Eduardo, la mère de Maria Jurandir a continué à s'occuper de cette dernière et de son frère ; elle a toujours été fière d'eux et ne les a jamais cachés aux autres. L'histoire de Maria Jurandir n'est donc qu'en partie similaire à celle d'Eduardo. On peut en outre relever que Maria Jurandir a développé un sentiment de fierté à l'égard de ce qu'elle est : non pas une simple chouette, insiste-t-elle, mais bien mieux : un hibou jacurutu. C'est ce qui l'amène aussi à faire preuve d'un certain mépris pour les autres animaux avec lesquels Eduardo s'est lié d'amitié, notamment le crapaud Bolitrô, qu'elle qualifie de « petit crapaud anémique de rien du tout » (Ibid. 73). Autrement dit, Maria Jurandir, dont le passé peut être rapproché de celui d'Eduardo, a développé sa personnalité dans une direction opposée.

Jean Péloueyre et Eduardo évoluent au milieu d'un environnement qui les rejette pour ce qu'ils sont en apparence. Il leur faut, pour combattre leur souffrance et leur solitude, s'évader par la vie intérieure ou l'imagination, comme nous allons le voir. 
BIDAUD

\section{Souffrance et évasion}

Le thème de l'évasion est présent dans le roman de Mauriac aussi bien que dans celui de José Mauro de Vasconcelos. Face à un milieu qui les isole et ne les comprend pas, mais aussi face à la maladie, Jean et Eduardo développent une grande capacité à s'échapper, par la religion pour Jean, par l'imaginaire pour Eduardo.

Dans Le Baiser au lépreux, Jean, avant qu'il ne rencontre Noémi, est seul : tout le village se moque de lui ; sa tante et son cousin, Félicité et Fernand Cazenave, le méprisent ; et son père, $M$. Jérôme, hypocondriaque qui fait penser à la tante Léonie de Proust, l'aime certes mais est uniquement centré sur luimême. Quant à sa mère, Jean l'a perdue alors qu'il était jeune ${ }^{3}$. Enfin, « (s)es trois années de collège, il [= Jean] les avait consumées en amitiés jalousement cachées : ni ce camarade Daniel Trasis, ni cet abbé maître de rhétorique, ne comprirent ses regards de chien perdu » (Mauriac 1969 : 19). Qui, parmi toutes ces personnes, pourrait être proche de Jean?

La religion est la seule chose qui permette à ce dernier de goûter un peu de joie et de sérénité. Jean aime ainsi se recueillir dans la paix de l'église le soir. Cette religion n'est pas tant, comme celle de son père, fondée sur la théologie, que sur les sentiments. Néanmoins, la foi de Jean fait l'objet d'un drame, qui survient quand il tombe sur des Morceaux choisis de Nietzsche qui le bouleversent :

Alors Jean Péloueyre lut ceci : «Qu'est-ce qui est bon ? - Tout ce qui exalte en l'homme le sentiment de puissance, la volonté de puissance, la puissance ellemême. Qu'est-ce qui est mauvais ? - Tout ce qui a sa racine dans la faiblesse. Périssent les faibles et les ratés : et qu'on les aide encore à disparaître ! Qu'estce qui est plus nuisible que n'importe quel vice ? - La pitié qu'éprouve l'action pour les déclassés et les faibles : le christianisme. »

Jean Péloueyre posa le livre ; ces paroles entraient en lui comme dans une chambre, dont on pousse les volets, l'embrasement d'un après-midi. (Ibid. 17)

3 Cette absence de la mère dans les premiers romans avant 1923 (date de publication de Génitrix) a par exemple été soulignée par J. E. Flower : « De tous les protagonistes principaux dans ces œuvres, ni Jean-Paul dans L’Enfant chargé de chaînes, ni le narrateur de Préséances, ni Jean Péloueyre dans Le Baiser au lépreux, n’a connu sa mère » (Flower 1995 : 9). 
Les phrases de Nietzsche poursuivent Jean tout au long du roman ; tout au long du roman, il s'interroge sur les maîtres et les esclaves ${ }^{4}$. De quel côté est Jean ? Il réexamine sa vie au prisme de l'aphorisme 260 de Par-delà le bien et le mal, celui, nous dit Mauriac, « qui a trait aux deux morales : celle des maîtres et celle des esclaves » (Ibid. 19) :

Un instant, il crut voir à ses pieds, pareille à un chêne déraciné, sa Foi. Sa Foi n'était-elle pas là, gisante, dans ce torride jour ? Non, non : l'arbre l'étreignait encore de ses mille racines ; après cette rafale, Jean Péloueyre en retrouvait dans son cœur l'ombre aimée, le mystère sous ces frondaisons drues et de nouveau immobiles. Mais il découvrait soudain que la Religion lui fut surtout un refuge. Au laideron orphelin, elle avait ouvert une nuit consolatrice. Quelqu'un sur l'autel tenait la place des amis qu'il n'avait pas eus, et la Vierge héritait de cette dévotion qu'il eût vouée à sa mère selon la chair ${ }^{5}$. (Ibid. 19-20)

Or la libération de Jean se produit à Paris, où il découvre une forme de laideur existentielle répandue sur tous les visages, comme nous l'avons vu plus haut, ce qui l'amène à comprendre qu'il n'y a aucun maître parmi les gens qui l'entourent. Surtout, positivement, il fait l'expérience fondamentale de la révélation de l'amour après avoir fui brusquement de la chambre où il avait suivi une prostituée, avec qui il n'a finalement eu aucune relation. Ce que Jean découvre alors, c'est la joie de l'amour à travers la fidélité à Noémi, une joie qui est élevée à un état divin à travers l'idée de l'immortalité de l'amour :

Il ne souffrait plus ; tout était pur en lui. Il se délectait de sa misère sans souillure. Noémi et Jean s'aimeraient dans un jour d'été sans déclin. D’avance il goûta l'accord de leur chair glorifiée. O lumière où s'appelleront leurs corps immortels, leurs corps incorruptibles! Jean Péloueyre dit à voix haute : « Il n'est pas de Maîtres, nous naissons tous esclaves et nous devenons vos affranchis, Seigneur ». (Ibid. 111)

Il n'est pas de maîtres ni d'esclaves, mais des hommes libres parce que capables d'aimer, parce que voulant aimer. C'est l'amour qui délivre en même temps qu'il lie, ou plutôt qu'il relie. Et c'est d'aimer et de se sentir aimé qui conduira Jean à pouvoir faire l'acte de charité qu'il accomplit à la fin du roman,

4 Muthia Aisha Chandra (2012) voit dans le conflit entre Nietzsche et la foi de Jean l'opposition du libre-arbitre et du jansénisme, Jean se défaisant progressivement de ce dernier qui est présent chez son père.

5 On pourra également se reporter, sur la religion comme besoin chez l'enfant dans l'œuvre de Mauriac, à Raymond Brazeau (1963 : 104 et suiv.). 
lorsqu' il va passer ses après-midis chez le fils du docteur Pieuchon atteint de la tuberculose et finit par mourir.

Dans $O$ Veleiro de Cristal, Eduardo réinvente l'univers où il se trouve afin de s'évader. Comme Zezé, le narrateur de O Meu Pé de Laranja Lima (2011 [1968], français « Mon bel oranger ») et de Vamos Aquecer O Sol (1977 [1974], français «Allons réveiller le soleil »), il a la capacité de s'émerveiller et de transformer ce qui l'entoure par l'imagination. Dès le début, lorsqu'il découvre la villa où il va séjourner avec sa tante et qu'il la voit se déployer dans la nuit avec toutes ses lumières, il apparente cette dernière à un voilier de cristal, qui donnera son titre au récit. Toute la maison est dès lors reconstruite par Eduardo à l'image d'un vrai voilier, qui représente une métaphore du voyage à travers l'imagination.

La particularité du Voilier est d'être peuplé d'animaux qui s'animent. Il y a d'abord la statue du tigre chinois Gakusha, dont Eduardo découvre qu'elle contient un tigre vivant après qu'il a posé son oreille contre le corps du tigre de bronze et que Gakusha a commencé à lui parler. Il y a, aussi, Maria Jurandir la chouette et Bolitrô le crapaud. Tous ces animaux deviennent les amis d'Eduardo, qui s'enquiert de l'histoire de leur vie comme eux de la sienne. Ils lui permettent d'échapper à la maladie et la tristesse. Une nuit, lui qui est incapable de se déplacer fait notamment grâce à Gakusha un voyage merveilleux qui lui permet de découvrir des paysages éblouissants. Tout cet univers est chargé de poésie. Ainsi lorsqu'Eduardo et Gakusha se trouvent au milieu de la mer et qu'Eduardo touche cette dernière : « Il glissait sa main dans l'eau, faisait des roses d'écume d'un blanc limpide et les jetait en l'air comme s'il avait offert des fleurs à la lune » (Vasconcelos $1973: 65$ ).

Eduardo s'émerveille devant toute la beauté de la nature, de la mer et des créatures. Il s'émerveille, aussi, devant les noms et leurs sonorités, qui le font rêver. Les mots l'enchantent : lorsqu'il entend Maria Jurandir lui déclarer qu'elle n'est pas une chouette mais un hibou jacurutu, ou qu'elle lui parle de l'arbre où elle est née, un mirindiba, Eduardo rêve sur ces sonorités mystérieuses et exotiques qui lui évoquent la forêt vierge.

Le thème des noms est important dans $O$ Veleiro de Cristal, comme ailleurs dans l'œuvre de José Mauro de Vasconcelos. Quand Eduardo entre en relation avec les animaux du Voilier pour la première fois, leur nom fait souvent l'objet d'un questionnement et éventuellement d'une réinvention. Lors de la première rencontre d'Eduardo et de Gakusha, comme Eduardo demande à ce dernier comment il s'appelle, Gakusha lui explique que son maitre l'appelle Chinois ou Gakusha. Le nom du tigre fait aussitôt l'objet d'un éclaircissement par l'étymologie (Gakusha signifie «sage » en japonais), mais comme Eduardo s'avère incapable de le prononcer correctement, il se trouve transformé en 
un prénom qui commence par la même syllabe « ga », Gabriel, prénom qui plaît à Eduardo car c'est celui de l'ange qui annonce la naissance de Jésus à la Vierge Marie. Un peu plus tard, lorsqu'il rencontre Bolitrô le crapaud, Eduardo se renseigne sur l'origine du prénom de ce dernier, derrière lequel se cache une histoire - en réalité, Bolitrô s'appelait à sa naissance Innocence, mais il a préféré changer de nom après s'être entiché de celui d'un ministre français. Il s'est littéralement approprié son nom : pour lui, ce n'est pas lui qui a un nom de ministre, mais le ministre qui a un nom de crapaud. Bolitrô mentionne également à Eduardo son amie serpente-religieuse Dona Janirana, mais avoue qu'il n'a aucune idée quant à l'origine de son nom. Il s'agit alors pour Eduardo de rêver sur celui-ci en le remotivant : «Moi, je tire plus ou moins mes conclusions. Elle est gentille, c'est pour cette raison qu'on entend Anna à la fin de son nom. Anna aussi a une âme de religieuse » (Ibid. 57). Dona Maria Jurandir, la chouette, a également changé de prénom : sa mère l'avait appelée Mintaka, mais elle trouvait ce prénom affreux et a préféré prendre celui d'une femme dont elle a lu dans les journaux qu'elle avait été assassinée de mille sept cents cinquante-deux coups de couteau - Maria Jurandir étant portée sur le tragique et n'aimant que les choses tristes. Tous les noms des animaux ont une histoire, et plusieurs d'entre eux en ont pris de nouveaux car ils n'aimaient pas ceux qu'ils avaient au début, comme le raconte Bolitrô sur un ton de secret à Eduardo. Pour Eduardo, le nom est donc un mystère à lire ou à réinventer et fait partie de son univers enchanté.

Le monde magique que découvre Eduardo est par ailleurs valorisé dans son opposition au monde physique. Gakusha est un tigre merveilleux, non pas en tant que tigre qu'il a été auparavant (sa vie, comme il l'affirme à Eduardo, n'a rien d'extraordinaire ; il a été très jeune enlevé à la forêt où il vivait pour être élevé comme tigre domestique), mais en tant que tigre qui parle avec Eduardo, qui l'aime et le fait voyager.

Or quel est le « vrai » monde ? Celui où Eduardo s'évade ou celui des autres hommes ? La question se pose à la fin du livre, quand Anna confie à Eduardo, qui est sur le point de mourir, qu'elle l'emmènera dans la maison d'un ami où ils feront un voyage ensemble ${ }^{6}$. Eduardo, en effet, dit à Anna qu'il connaît cette maison et qu'ils y sont déjà allés, et il se met à lui décrire le Voilier de Cristal que nous avons connu tout au long du récit et qui correspond point par point avec la maison à laquelle pense Anna. Mais Eduardo et elle ne s'y sont jamais rendus

6 On pourra également se reporter à l'article de Penha Lucilda de Souza Silvestre, qui explore une problématique similaire en ce qui concerne Rosinha, Minha Canoa [traduction française Rosinha mon canoë] de José Mauro de Vasconcelos. 
BIDAUD

en réalité ; il est donc impossible qu’Eduardo connaisse le Voilier de Cristal. Comment le connaît-il ? En fait, le lecteur découvre que tout le récit qu'il a lu n'est peut-être que l'imagination d'Eduardo, mais en même temps cette imagination est donnée comme plus vraie que la réalité : puisqu'il est capable de décrire précisément ce Voilier où il n'a jamais été, c'est bien qu'Eduardo le connaît. Le caractère fictif du monde de ce dernier est ainsi remis en question à la fin du livre et les points de vue se trouvent inversés. Le rêve d'Eduardo devient la réalité ; la bonté qu'il a découverte appartient à un monde plus vrai que celui des hommes. Et Anna finit par croire au monde d'Eduardo, comme on le voit dans sa prière poignante à la fin du récit :

Ses jambes fléchirent et elle s'agenouilla en appuyant sa tête sur la fenêtre comme une enfant sans défense. Et ses lèvres s'entrouvrirent dans une aspiration de désirs et de prière.

- Continue ton beau voyage, mon cœur. Continue sur ton Voilier de Cristal, sur ton voilier d'étoiles, vers un monde de silence et de paix !

- Maintenant je sais que tu t'en vas, mon cœur !

- Accompagné de ton tigre, de ton crapaud, de ta chouette et de tant d'autres petites créatures comme toi, que ton cœur a toujours aimées.

- Comme tu es déjà loin. Est-ce que les étoiles sont plus belles vues de près ? (Ibid. 92)

Monde d'étoiles triste et poignant, monde de souffrance, O Veleiro de Cristal est aussi un livre sur la force du rêve et de la foi - une prière brûlante d'amour.

\section{La Charité}

Le thème de la charité est fondamental dans Le Baiser au lépreux comme dans O Veleiro de Cristal.

Le titre même du livre de Mauriac, Le Baiser au lépreux, évoque la charité à travers la métaphore de la lèpre, en même temps qu'il mentionne ce qui la guérit - le baiser qu'elle donne. Un tel titre fait songer à L'Annonce faite à Marie de Claudel et à la scène d'ouverture de cette pièce, où Violaine embrasse Pierre de Craon tout en sachant qu'il a la lèpre (Claudel 1972 [1948]). De la même façon, Noémi embrasse Jean malgré la répulsion physique qu'elle éprouve pour lui. Pourtant, si la notion de charité telle que le titre la signifie semble renvoyer au premier abord à Noémi exclusivement, il y a lieu de constater qu'elle est en réalité double dans Le Baiser au lépreux et qu'elle croît progressivement durant le roman chez Noémi comme chez Jean. Les deux époux font en effet preuve d'une charité de plus en plus grande l'un envers l'autre, dépassant 
ainsi la dimension corporelle de l'amour pour arriver à l'amour véritablement chrétien - celui de deux âmes qui se fondent l'une dans l'autre à travers la charité réciproque mais également, selon la perspective chrétienne, à travers l'ouverture de cette charité au monde.

$\mathrm{Au}$ début du récit, le mariage de Jean et Noémi n'est pas, pour la jeune femme, un mariage d'amour, mais d'abord un mariage que ses parents et le curé ont décidé pour elle. Noémi est présentée comme une jeune fille simple et religieuse, toute tournée vers Dieu. Malgré la peur que ce mariage lui inspire à cause de l'aspect physique de Jean, ce qui la pousse ponctuellement à vouloir entrer au couvent, elle accepte finalement son époux par devoir (Mauriac 1969 : 57). Physiquement, Noémi éprouve de la répugnance pour Jean, mais, chrétiennement, elle veut l'aimer. Aussi durant la majeure partie du récit se reproche-t-elle en même temps son dégoût et de n'être pas une bonne épouse selon Dieu, comme l'écrit Mauriac. Elle fait tous les efforts qu'elle peut pour aller vers son mari : elle s'oblige à l'embrasser, à le retenir au moment où il « décide » de partir pour Paris, etc. Il ne s'agit pas toutefois simplement pour Noémi de respecter des devoirs d'une façon purement formelle ; elle veut vraiment aimer Jean. Quant à ce dernier, il est conscient que son aspect inspire à Noémi un dégoût qu'elle combat : aussi fuit-il le plus souvent qu'il lui est possible et fait-il semblant de dormir lorsqu'elle l'embrasse. Il ne la contraint pas, ne cherche pas à l'approcher physiquement. Les deux époux tels que Mauriac nous les décrit redoublent de zèle l'un pour l'autre :

Chacun veillait à ne pas toucher la blessure de l'autre. Leurs gestes furent mesurés pour se faire moins souffrir : quand Noémi se déshabillait, il [= Jean] regardait ailleurs et n'entrait jamais dans le cabinet de toilette quand elle s'y lavait. [...] Jamais ils n'échangèrent un reproche même muet, mais d'un regard se demandaient l'un à l'autre pardon. Ils décidèrent de réciter ensemble leur prière : ennemis dans la chair, ils s'unissaient dans cette imploration du soir ; leurs voix au moins pouvaient se confondre ; côte à côte et séparés, ils se rejoignaient à l'infini. (Ibid. 87-88)

La première partie du récit, jusqu'au départ de Jean pour Paris, est fondée sur ce sentiment de respect mutuel des deux époux. La deuxième partie, qui se situe au moment où Jean part pour la capitale, laisse place à une nouvelle forme de charité qui sera de plus en plus forte.

L'éloignement entre Noémi et Jean, en même temps qu'il les sépare physiquement, leur permet de découvrir leur amour dans l'épreuve. Le passage déjà mentionné, où Jean accompagne une prostituée avant de fuir, lui révèle le lien immatériel qui l'attache à Noémi : loin d'elle, alors qu'il est cette fois 
BIDAUD

considéré comme quelqu'un qui n'inspire aucun dégoût particulier dans ce Paris où personne ne le remarque, il refuse l'amour physique que Noémi a refusé pour lui. Il en va de même avec Noémi. Durant l'absence de Jean, en effet, arrive un troisième personnage, le jeune docteur qui vient soigner M. Jérôme, puis Jean lorsque ce dernier est de retour et qu'il tombe malade. Or le jeune docteur voudrait séduire Noémi et celle-ci découvre qu'elle éprouve également de l'attirance pour lui. Le rapport qui existe entre le jeune médecin, Noémi et Jean fait penser à Madame Bovary et au trio Rodolphe, Emma et Charles. Mais, alors que, dans Madame Bovary, Emma est séduite par Rodolphe, Noémi fuit le jeune médecin qui cherche à avoir une aventure avec elle en combattant de toutes ses forces l'attirance qu'elle ressent et en le repoussant, par fidélité pour Jean.

La dernière partie du récit donne enfin à lire la charité qui resplendit depuis le couple jusqu'aux autres. Lorsque Jean va voir Robert, le fils du docteur Pieuchon tombé malade, il connaît les risques qu'il court. Et pourtant, il va passer tous les après-midis avec ce jeune homme atteint de la tuberculose. Il revêt alors une dimension christique ${ }^{7}$ que le curé comprend :

Le prêtre concevait qu'il y avait loin du petit être chétif, de ce Jean Péloueyre à peine capable, aux veilles de grandes fêtes, de frotter les cristaux des lustres et de ramasser les longues mousses dont les dames faisaient des guirlandes, qu'il y avait loin du tueur de pies à ce mourant qui donnait sa vie pour le salut de plusieurs. Le curé s'abîmait devant Celui dont le secret est de rendre semblables à Dieu, des esclaves. (Ibid. 155-156)

Le Baiser au lépreux devient alors le baiser $d u$ lépreux : la charité dont Noémi fait preuve avec lui, Jean la lui rend au centuple, à elle et aux autres, comprenant qu'il a été aimé : « Croyait-il maintenant qu'elle l'aimait ? Il le croyait et disait : "Soyez béni à jamais, mon Dieu, qui, avant que je meure, m'avez donné l'amour d'une femme...” » (Ibid. 149). Cet amour qui se donne à l'autre se répercute sur Noémi, qui passe ainsi, au cours du roman, du devoir d'épouse à la charité

Cette dimension est évoquée précédemment, la nuit où Jean est malade et souffre en même temps qu'il s'efforce de ne rien laisser voir à Noémi : « Ayant allumé une bougie, elle vit qu'il était trempé de sueur. Elle le regardait avec angoisse. Les yeux clos, il paraissait attentif à un travail mystérieux en lui. Il sourit à sa femme, et Noémi fut bouleversée par ce sourire si tendre, si calme. Et il dit à mi-voix : "J'ai soif” 》 (Mauriac 1969 : 141). Les paroles de Jean, «J’ai soif », reprennent celles de Jésus sur la croix. 
vraie, libre et consentie dans la fidélité à Jean, ce qu'expriment magnifiquement les dernières phrases du livre :

Cette bourgeoise un peu épaisse ne pouvait pas ne pas se dépasser elle-même : toute route lui était fermée, hors le renoncement. Dès cette minute-là, dans la pignada pleine de mouches, elle connut que sa fidélité au mort serait son humble gloire et qu'il ne lui appartenait pas de s'y soustraire. Ainsi courut Noémi à travers les brandes, jusqu'à ce qu'épuisée, les souliers lourds de sable, elle dût enserrer un chêne rabougri sous la bure de ses feuilles mortes mais toutes frémissantes d'un souffle de feu, - un chêne noir qui ressemblait à Jean Péloueyre. (Ibid. 177-178)

La charité anime également $O$ Veleiro de Cristal, où l'on rencontre, tout d'abord, la charité des animaux pour Eduardo. Tous parlent avec lui, de Gakusha à Bolitrô en passant par Maria Jurandir. Gakusha, notamment, ressent une très grande affection pour Eduardo et veut lui donner le bonheur de voyager et de découvrir des paysages merveilleux, pour qu'Eduardo, au moins une fois dans sa vie, connaisse cette joie. Il désire non seulement emmener Eduardo où ce dernier souhaite aller, mais aussi lui faire partager sa propre vie en lui montrant ce qu'il appelle «son lac ». Gakusha se révèle ainsi pour Eduardo un ami, un frère, davantage que ses frères Marcelo et Sérgio, puisqu'il l'accueille et l'aime tel qu'il est, ainsi que le constate Eduardo.

Parmi tous les animaux qui habitent le Voilier de Cristal, l'un d'entre eux est nommé mais n'apparaît pas. Il s'agit de Dona Janirana, la serpentereligieuse dont parle Bolitrô. Cette dernière a décidé de se retirer du monde pour vivre cachée et prier pour les autres, comme l'explique Bolitrô à Eduardo (Vasconcelos 1973 : 55). Celui-ci compare alors Dona Janirana à Anna ; comme Anna, Dona Janirana a renoncé aux gloires du monde terrestre pour se consacrer entièrement, dans son cas, à Dieu et aux autres.

Si Dona Janirana est rapprochée d'Anna, on peut également la rapprocher d'Eduardo. Comme Eduardo, en effet, elle vit cachée, et comme Eduardo, elle suscite a priori, par sa réputation, une forme de dégoût. Eduardo s'étonne d'abord que Bolitrô puisse être ami avec elle, mais le crapaud corrige ses préjugés :

- Attendez un peu, Bolitrô, vous m'étonnez. Vous avez bien dit une serpente ?

- Exactement.

- Mais est-ce que les serpents ne mangent pas les crapauds ?

- Ce sont des légendes. Tous les serpents ne mangent pas les crapauds.

(Ibid. 54-55) 
BIDAUD

L'âme d'Eduardo est belle, comme celle de Dona Janirana ; tous deux souffrent de leur image, physique pour Eduardo, morale pour Dona Janirana, apparentée, en tant que serpente, à une forme de mal, alors qu'elle est la charité même.

L'équivalent de Dona Janirana, comme l'onomastique nous invite à l'interpréter, est, dans le domaine des humains, Anna. Comme Noémi qui passe du devoir à la charité dans Le Baiser au lépreux, Anna a connu une conversion dans sa vie. Celle-ci a eu lieu bien plus tôt, comme elle le confie à la femme du jardinier de la villa un jour où toutes deux se trouvent dans le jardin - et c'est à Eduardo qu'elle le doit, c'est à lui qu'elle doit d'avoir retrouvé l'amour pour le prochain et de s'être décentrée d'elle-même. C'est dans la contemplation de la faiblesse d'Eduardo, de la fragilité de cet être mal-aimé et rejeté de tous, qu'elle a retrouvé la possibilité d'aimer. Elle aurait pu et pourrait avoir une vie complètement autre (comme le lui dit Maria, la femme du jardinier, elle est encore très belle), mais elle a décidé de prendre soin d'Eduardo. Ce dernier affirme ainsi à Gakusha : «La tendresse de son grand cœur l'a convaincue que je suis tout dans sa vie. Pour moi c'est beaucoup, mais pour une personne comme Anna c'est peu demander à la vie $\gg$ (Ibid. 45). Le lien qui attache Anna à son neveu est presque fusionnel : alors qu'Eduardo attend en vain que sa mère l'appelle ou vienne le voir, même sur son lit de mort, il n'y a qu'Anna qui soit proche de lui et qui l'aime, comme il le lui confie avant de mourir.

Si Anna prend soin d'Eduardo tout au long du roman, c'est Eduardo qui, alors qu'il est en train de mourir, la bouleverse par la révélation qu'il lui fait du Voilier de Cristal et la fait accéder à une dimension apparemment surnaturelle ; c'est lui qui, devant la mort qui arrive, la rassure : Anna, finalement, voit elle aussi à la fin et comprend toute la force de l'imagination et de la foi ; elle comprend qu'Eduardo est à présent sur son Voilier avec tous les animaux qui l'habitent et c'est elle qui lui demande son aide :

Pour la dernière fois elle joignit les mains et supplia :

- Mon chéri, quand tu atteindras la beauté des étoiles, quand tu toucheras leur éclat à toutes, n'oublie pas... Envoie-moi une goutte de tendresse, un rayon d'amour, pour que mes bras n'étreignent plus ton absence et que mon cœur cesse de marcher pour toujours dans le désespoir !... (Ibid. 93) 
Ubi caritas et amor

\title{
Conclusion
}

Le Baiser au lépreux et $O$ Veleiro de Cristal présentent de profondes similitudes, à la fois du point de vue de l'histoire qu'ils racontent et de la charité qu'ils incarnent. En peignant des personnages rejetés pour leur image, ils mettent tous deux en scène le thème de la laideur extérieure à laquelle s'oppose la beauté intérieure. La charité des personnages les uns pour les autres renvoie à une vision de l'amour comme agapè dans Le Baiser au lépreux comme dans $O$ Veleiro de Cristal. Un amour qui transcende le visible et se révèle plus fort que la mort, à l'instar de l'amour auquel invite saint Paul dans la Première Lettre aux Corinthiens.

Au milieu de toute la tristesse dont ils vibrent, les deux récits, Le Baiser au lépreux et $O$ Veleiro de Cristal, sont pleins d'une chaude bonté qui réchauffe l'âme et le cœur.

\author{
Samuel Bidaud \\ bidaudsamuel@gmail.com \\ Univerzita Palackého v Olomouci \\ Neředín 4,804 \\ U Letiště 847 \\ 77900 Olomouc \\ ČESKA REPUBLIKA / RÉPUBLIQUE TCHÈQUE
}

\section{Bibliographie}

Brazeau, R. 1963 [2011 pour la numérisation]. La physionomie religieuse de l'enfant dans les romans de François Mauriac. Thèse présentée à la Faculté des Arts de l'Université d'Ottawa en vue de l'obtention de la Maîtrise ès Arts, ruor.uottawa.ca/ bitstream/10393/22495/1/EC56246.PDF. [Dernière consultation en avril 2020.]

Chandra, M. A. 2012. Jansenism Thought in François Mauriac's Novel: Le Baiser au Lépreux. - Procedia - Social and Behavioral Sciences, 65/3, 338-343. https://doi. org/10.1016/j.sbspro.2012.11.131

Claudel, P. 1972 [1948]. L'Annonce faite à Marie. Paris : Gallimard, collection « Folio ».

Flower, J. E. 1995. La Mère, personnage clé dans les romans de Mauriac. - J. E. Flower, éd., François Mauriac. Psycholectures - Psychoreadings. Exeter : University of Exeter Press ; Bordeaux : Presses Universitaires de Bordeaux, 9-20.

Mauriac, F. 1969 [1922]. Le Baiser au lépreux. Paris : Bernard Grasset.

Souza Silvestre, P. L. (de). 2014. O normal e o patológico: uma leitura de Rosinha, Minha Canoa de José Mauro De Vasconcelos (Diálogo entre literatura e psicologia). - CLARABOIA: Revista do Curso de Letras da UENP, 1/1, 78-91. 
BIDAUD

Takayama, T. 2001. Mauriac lecteur de Balzac. L’Année balzacienne, 1/2, 305-316. Vasconcelos, J. M. (de) 1973. O Veleiro de Cristal. São Paulo : Melhoramentos. Vasconcelos, J. M. (de) 2011 [1968]. O Meu Pé de Laranja Lima. Lisboa : Dinapress.

Vasconcelos, J. M. (de) 1977 [1974]. Vamos Aquecer O Sol. São Paulo : Melhoramentos. 